\title{
Introducing fractal dimension algorithms to calculate the Hurst exponent of financial time series
}

\author{
M.J. Sánchez-Granero ${ }^{1}$, M. Fernández-Martínez ${ }^{1}$, and J.E. Trinidad-Segovia ${ }^{2, a}$ \\ 1 Area of Geometry and Topology, Faculty of Science, Universidad de Almería, 04120 Almería, Spain \\ 2 Department of Accounting and Finance, Faculty of Economics and Business, Universidad de Almería, 04120 Almería, Spain
}

Received 30 September 2011 / Received in final form 24 December 2011

Published online 5 March 2012 - (c) EDP Sciences, Società Italiana di Fisica, Springer-Verlag 2012

\begin{abstract}
In this paper, three new algorithms are introduced in order to explore long memory in financial time series. They are based on a new concept of fractal dimension of a curve. A mathematical support is provided for each algorithm and its accuracy is tested for different length time series by Monte Carlo simulations. In particular, in the case of short length series, the introduced algorithms perform much better than the classical methods. Finally, an empirical application for some stock market indexes as well as some individual stocks is presented.
\end{abstract}

\section{Introduction}

The discussion about the market efficiency is still, after half a century, a classical topic in finance. Indeed, nowadays researchers can be classified into two different tendencies: some of them consider that stock market prices follow a Brownian motion $^{1}$, which implies a certain level of randomness in the evolution of trends (see $[1,2]$ ), while others try to prove that prices reflect a fractional Brownian motion, which means that market shows a fractal behavior with long memory (see [3-7]). This second idea involves some interesting effects since the existence of memory in market prices evolution would imply that some extra information could be available in order to make investments.

In this way, this second school of thought was born after the publication of [8], which is one of the first Mandelbrot's works in economy. The main research of these authors is focused on the study of long memory processes in financial time series. Obviously, note that the detection of memory implies the rejection of the efficient market hypothesis (supported by $[9,10]$, for instance).

The classical procedure in order to test long memory in market series is based on the calculation of the Hurst exponent, which was first introduced by english hydrologist Hurst in 1951, and has been classically estimated through the next two procedures:

1. R/S analysis (see $[1,7,11-13])$;

2. DFA (detrended fluctuation analysis) (see for example, $[14-18])$.

\footnotetext{
a e-mail: jetrini@ual.es

1 In 1959, the concept of Brownian motion in finance was introduced by the astronomer Robert Osborne by comparing the stock market equilibrium with the equilibrium of particles in statistical mechanics.
}

The Hurst exponent, usually denoted by $H$, has to lie between 0 and 1 . Thus, when the process is a Brownian motion then the value of $H$ is equal to 0.5 . When it is persistent it will be greater than 0.5 , and when it is antipersistent that quantity will be less than 0.5 . Examples of extreme values 0 and 1 are $1 / f$ noise and a simple linear trend, respectively.

As use to be normal in social sciences, most of the quantitative models are being extended from other scientific areas, such as statistical physics, mathematics and natural sciences (see [19]): in this sense, both R/S analysis and DFA do not provide an exception. Under the theory that the previous models and instruments need an adaptation before being exported, in a previous paper (see [20]) the authors proved that the classical methods to estimate the Hurst exponent do not work properly in short series, so they proposed a new methodology, which is based on two geometrical approaches named GM1 and GM2, that, in particular, solve this problem.

During last years new ways to explore market memory have been developed: several of them based on the calculation of fractal dimension, such as [21-23], to quote some of them. Nevertheless, their approach is totally different from ours.

In this paper, we will introduce some new procedures in order to explore market memory, based on a new concept of fractal dimension, which will be called FD methods. Their behavior and accuracy are tested from different length time series using Monte Carlo simulations. Moreover, a mathematical support is provided for each algorithm and we also apply them to explore long memory in financial time series.

The organization of the paper is as follows: in Section 2 we present the concept as well as the mathematical 
bases of a fractal structure. In Section 3, by using fractal structures, we introduce a new concept of fractal dimension particularly appropriated for the image of any curve. Section 4 contains the main theoretical results as well as the mathematical formulation of our algorithms to estimate the Hurst exponent. The accuracy of FD methods is tested in detail in Section 5, where a comparison between geometrical, classical and fractal approaches is provided. The paper continues with empirical applications to the analysis of long memory in stock market indexes and some individual stocks, respectively. Finally, Section 7 contains the main conclusions.

\section{Exploring the complexity of curves using fractal structures}

Since Benoît Mandelbrot introduced the concept of fractal set ([24]), this kind of non-linear objects have received attention from a wide range of science fields, including social sciences. In this way, [25] and its references contain a detailed selection of such applications.

The main tool we provide in order to describe fractals from a rigorous point of view is the concept of fractal structure, which was first sketched in [26], and then used in [27] to characterize non-archimedean quasimetrization, though a more natural use of it is in the study of fractals. A fractal structure is just a countable family of coverings, called levels, of a given set which approaches the space. More information is provided about the space when deeper levels in its fractal structure are explored. For instance, note that some kind of common fractal objects like self-similar sets are equipped with a fractal structure in a natural way (see [28], Def. 4.4) as well as it happens with any euclidean space $R^{d}$ which also has a natural fractal structure consisting of $d$-dimensional cubes of side $\frac{1}{2^{n}}$ on the $n$ th-level of its fractal structure (see [29], Def. 3.1). Accordingly, fractal structures provide a rigorous description of these non-classical objects and lead to formalize the definition of a fractal from a mathematical point of view.

Note that [30] contains a wide range of applications of fractal structures in some mathematical research areas. In this way, space-filling curves, topological and fractal dimensions, self-similar sets, metrization, non-archimedean quasimetrization and transitive quasiuniformities are some examples, just to name a few.

Next we introduce some preliminary concepts which are necessary in order to define a fractal structure on any set $X$. Indeed, let $\Gamma_{1}$ and $\Gamma_{2}$ be two coverings of $X$ (a family $\Gamma$ of subsets of $X$ is said to be a covering if $X=$ $\left.\cup_{A \in \Gamma} A\right)$. Then, we will write $\Gamma_{1} \prec \Gamma_{2}$ to denote the fact that $\Gamma_{1}$ is a refinement of $\Gamma_{2}$, that is, for all $A \in \Gamma_{1}$ there exists $B \in \Gamma_{2}$ such that $A \subseteq B$. Similarly, $\Gamma_{1} \prec \prec \Gamma_{2}$ means that $\Gamma_{1} \prec \Gamma_{2}$, and for all $B \in \Gamma_{2}$ it is verified that $B=\cup\left\{A \in \Gamma_{1}: A \subseteq B\right\}$.

A fractal structure on a set $X$ is defined as a countable family of coverings of $X$, namely, $\boldsymbol{\Gamma}=\left\{\Gamma_{n}: n \in N\right\}$, such that $\Gamma_{n+1} \prec \prec \Gamma_{n}$ for each $n \in N$.
Fractal structures provide a powerful tool in order to study the complexity of a given set by means of its fractal dimension value. In this paper, we develop a new definition of fractal dimension to be used to distinguish and classify different kinds of processes such as Brownian motions (resp. fractional Brownian motions), whose relevance consists of its usefulness in order to modelize random processes (resp. long memory processes) in time series. These motions have been widely applied to the analysis of financial time series.

One of our goals is to define a fractal structure on the image set of a real curve. Thus, for irregular curves, our fractal dimension definition will provide some interesting information about its structure and pattern. Indeed, if $\alpha$ : $I \rightarrow \mathbb{R}$ is a parametrization of a real curve, where $I$ is a subinterval of the real line which has a fractal structure $\boldsymbol{\Gamma}$, then it is always possible to define an induced fractal structure $\boldsymbol{\Delta}$ on its image set $\alpha(I)$. This idea allows to study the structure of any curve and leads to interesting analysis of time series like those used in finance.

The natural fractal structure on the real line (see [29]) is defined as the countable family of coverings $\boldsymbol{\Gamma}=\left\{\Gamma_{n}\right.$ : $n \in \mathbb{N}\}$, whose levels are given by $\Gamma_{n}=\left\{\left[\frac{k}{2^{n}}, \frac{k+1}{2^{n}}\right]: k \in \mathbb{Z}\right\}$ for all $n \in \mathbb{N}$. In particular, it is also possible to consider a natural fractal structure induced on real subsets from the previous one. For instance, the natural fractal structure induced on the interval $[0,1] \subset \mathbb{R}$ may be defined as the family of coverings $\boldsymbol{\Gamma}$, whose levels are $\Gamma_{n}=\left\{\left[\frac{k}{2^{n}}, \frac{k+1}{2^{n}}\right]\right.$ : $\left.k \in\left\{0,1, \ldots, 2^{n}-1\right\}\right\}$ for all natural number $n$. We can define now the fractal structure induced in the image of a curve.

Definition 1. Let $\alpha: I \rightarrow \mathbb{R}$ be a parametrization of a real curve where $I \subset \mathbb{R}$ is a closed real subinterval and let $\boldsymbol{\Gamma}$ be the natural fractal structure on $I$. Then, the fractal structure induced by $\boldsymbol{\Gamma}$ in the image set $\alpha(I) \subset \mathbb{R}$ is defined as the countable family of coverings $\boldsymbol{\Delta}=\left\{\Delta_{n}: n \in\right.$ $\mathbb{N}$ \}, whose levels are given by

$$
\Delta_{n}=\alpha\left(\Gamma_{n}\right)=\left\{\alpha(A): A \in \Gamma_{n}\right\} .
$$

To conclude this section, Figure 1 illustrates the meaning of Definition 1. In this case, it is shown the first two levels of the fractal structure induced in the image of a given Brownian motion. In the bottom of the images, we can see the elements (horizontal bars on the bottom) of level 1 (in the first image) and 2 (in the second image) of $\boldsymbol{\Gamma}$. The image of those elements gives the elements (vertical bars on the left) of level 1 (first image) and 2 (second image) of $\boldsymbol{\Delta}$, which are shown in the left side of the images.

\section{The fractal dimension of a curve}

A new concept of fractal dimension for a curve is introduced in this section. The classical fractal dimension theory (box-counting dimension and Hausdorff dimension) deals with the dimension of a set while we define the dimension of the parametrization of a curve. So, different parametrizations lead to different values of the dimension. 

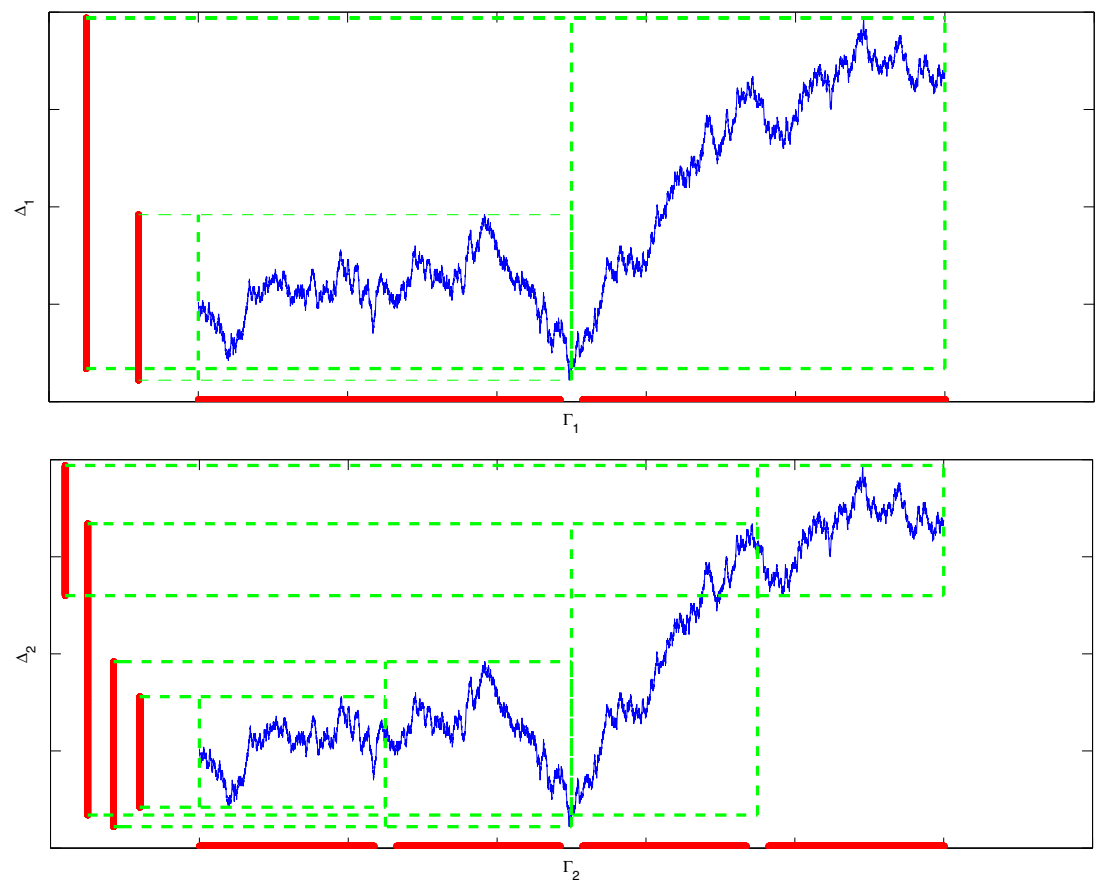

Fig. 1. (Color online) The two figures above show the graph of a Brownian motion $\alpha$ and the first two levels of its induced fractal structure $\boldsymbol{\Delta}$, namely $\Delta_{1}$ and $\Delta_{2}$. The horizontal lines at the bottom represent the elements of the natural fractal structure $\boldsymbol{\Gamma}$ defined on $I$, namely, $\Gamma_{1}$ and $\Gamma_{2}$. Furthermore, note that the image of each dotted line border box by $\alpha$ leads to the elements of $\boldsymbol{\Delta}$ (the vertical bars on the left).

This gives much more information than we get if we only calculate the dimension of the image of the curve and it is someway equivalent to calculate the dimension of the graph of the curve. We refer the reader to [31] for the study of the classical fractal dimension theory.

The next definition we provide is the key concept of the paper. It is an application of fractal dimension III defined in [32], and is based both in the box-counting and Hausdorff dimensions (see [31], end of Sect. 3.1 for the relation between both classical dimensions, which is also the motivation for this definition).

Definition 2. Let $\alpha: I \rightarrow \mathbb{R}$ be a parametrization of a real curve, where $I=[0,1]$ is the closed unit interval and let $\boldsymbol{\Gamma}$ be the natural fractal structure on $I$. Let also $\Delta$ be the fractal structure induced by $\boldsymbol{\Gamma}$ in the image set $\alpha(I) \subset \mathbb{R}$. Thus, for $s \geq 0$, consider the following sequence of quantities:

$$
\mathcal{H}_{n}^{s}(\alpha(I))=\sum\left\{\operatorname{diam}(B)^{s}: B \in \Delta_{n}\right\}
$$

for all $n \in \mathbb{N}$, where $\operatorname{diam}(B)$ is the diameter of $B$, that is, $\operatorname{diam}(B)=\sup \{|x-y|: x, y \in B\}$. Take also $\mathcal{H}^{s}(\alpha(I))=$ $\lim _{n \rightarrow \infty} \mathcal{H}_{n}^{s}(\alpha(I))$.

We define the fractal dimension of $\alpha$ with respect to $\Delta$, which will be denoted by $\operatorname{dim}_{\Delta}(\alpha)$, as one of the following equivalent quantities:

$$
\inf \left\{s: \mathcal{H}^{s}(\alpha(I))=0\right\}=\sup \left\{s: \mathcal{H}^{s}(\alpha(I))=\infty\right\} .
$$

Note that we are working with a very general concept of curve, since we do not require it to be continuous. Thus, in
Definition 2, the curve $\alpha$ does not need to be continuous. Also, it can be a time series and in particular, a financial time series. In this way, in the next section we will see that Definition 2 can be used as an alternative and also a generalization of the Hurst exponent. Thus, we study some new algorithms based on the calculation of the introduced fractal dimension, and in the particular case of (fractional) Brownian motions, we can determine the Hurst exponent by using such methods.

The interpretation of the values of the fractal dimension could be stated as follows. A bigger fractal dimension with $d \in[1, \infty)$ means that the oscillations of the curve increase at any scale. On the other hand, smaller values of $d$ imply a greater smoothness in the graph of the curve. In particular, if $\alpha$ is a smooth curve, then $\operatorname{dim}_{\Delta}(\alpha)=1$, and if $\alpha$ is a Brownian motion, then $\operatorname{dim}_{\Delta}(\alpha)=2$.

\section{Hurst exponent estimation using fractal dimension}

In this section, we will see how to use the fractal dimension introduced above to estimate the Hurst exponent. We can consider the fractal dimension as a generalization of the Hurst exponent, since it can be calculated for a wide range of motions, not only fractional Brownian motions. Anyway, instead of using the fractal dimension directly, we will use its relation with the Hurst exponent, since it is more familiar to researchers.

We present two kind of procedures to estimate $H$ by fractal dimension techniques. The first approach is to 
compute the fractal dimension by the ratio of the diameters of the elements of consecutive levels of $\boldsymbol{\Delta}$. The second approach is based on the relations of some statistical moments of consecutive levels of the induced fractal structure $\Delta$.

Thus, the structure of this section is as follows. First, we recall the basic properties of random functions and their increments. Then, we provide some theorems which give general conditions to calculate the fractal dimension and we introduce three new algorithms to calculate the fractal dimension based on those theorems. Finally, we also give some general conditions for a motion which allow to calculate the Hurst exponent as the inverse of the fractal dimension. In particular, this can be applied to (fractional) Brownian motions and (fractional) Lévy stable motions.

\subsection{Preliminaries: random functions and their increments. Self-affinity properties}

The definitions, properties and results that we recall next come from the theory of probability and stochastic processes and are essential in order to formalize our mathematical ideas. Some useful references are [23,31,33].

Let $(\mathbf{X}, \mathcal{A}, P)$ be a probability space and let $t \in[0, \infty)$ denote time. We say that $\mathbf{X}=\{X(t, \omega): t \geq 0\}$ is a random process or a random function from $[0, \infty) \times \Omega$ to $\mathbb{R}$, if $X(t, \omega)$ is a random variable for all $t \geq 0$ and all $\omega \in \Omega$ ( $\omega$ belongs to a sample space $\Omega$ ). We think of $\mathbf{X}$ as defining a sample function $t \mapsto X(t, \omega)$ for all $\omega \in \Omega$. Thus, the points of $\Omega$ parametrize the functions $\mathbf{X}:[0, \infty) \times \Omega \rightarrow \mathbb{R}$ and $P$ is a probability measure on this class of functions.

The definitions of a Brownian motion (BM for short), fractional Brownian motion (FBM), Lévy stable motion (LSM) and fractional Lévy stable motion (FLSM) can be found in [31] or [23].

Let $X(t, \omega)$ and $Y(t, \omega)$ be two random functions. The notation $X(t, \omega) \sim Y(t, \omega)$ means that the two preceding random functions have the same finite joint distribution functions. Recall also that

1. A random process $\mathbf{X}=\{X(t, \omega): t \geq 0\}$ is said to be $H$-self-similar if for some $H>0$,

$$
X(a t, \omega) \sim a^{H} X(t, \omega)
$$

for all $a>0$ and $t \geq 0$. The parameter $H$ is the selfsimilarity index or exponent.

2. The increments of a random function $X(t, \omega)$ are said to be:

(a) stationary, if for each $a>0$ and $t \geq 0$

$$
X(a+t, \omega)-X(a, \omega) \sim X(t, \omega)-X(0, \omega) ;
$$

(b) self-affine with parameter $H \geq 0$, if for any $h>0$ and any $t_{0} \geq 0$,

$$
\begin{aligned}
X\left(t_{0}\right. & +\tau, \omega)-X\left(t_{0}, \omega\right) \\
& \sim \frac{1}{h^{H}}\left\{X\left(t_{0}+h \tau, \omega\right)-X\left(t_{0}, \omega\right)\right\} .
\end{aligned}
$$

In this way, note that any FBM with exponent $H$ has stationary and self-affine increments with parameter $H$ (see [33], Thm. 3.3). On the other hand, note that by [33], Corollary 3.6 , we have that if a random function $X(t, \omega)$ has self-affine increments with parameter $H$, then a $T^{H}$ law as the following is satisfied:

$$
M(T, \omega) \sim T^{H} M(1, \omega)
$$

where its cumulative range is given by

$$
M(t, T, \omega)=\sup _{s \in[t, t+T]}\{Y(s, t, \omega)\}-\inf _{s \in[t, t+T]}\{Y(s, t, \omega)\}
$$

where $Y(s, t, \omega)=X(s, \omega)-X(t, \omega)$ and moreover, $M(T, \omega)=M(0, T, \omega)$. In particular, any FBM with parameter $H$ satisfies a $T^{H}$-law as shown in equation (4). Further, if $\mathbf{X}$ is a FBM, then one can replace sup and inf by max and min respectively, in equation (5) (see [33], Prop. 4.1).

Remark 1. Let $\alpha: I \rightarrow \mathbb{R}$ be a sample function of a random process $\mathbf{X}$ with stationary increments, where $I=$ $[0,1]$. Let $\boldsymbol{\Gamma}$ be the natural fractal structure on $I$ and $\boldsymbol{\Delta}$ the fractal structure induced by $\boldsymbol{\Gamma}$ in $\alpha(I)$. Then, for each $n \in \mathbb{N},\left\{\operatorname{diam}(A): A \in \Delta_{n}\right\}$ is a sample of the random variable $M\left(\frac{1}{2^{n}}, \omega\right)$.

\subsection{A first approximation to the fractal dimension: algorithm FD1}

We introduce a first algorithm to calculate the fractal dimension of a curve. First, we prove a result which is the base for the algorithm FD1. Then, we prove that for FBMs or FLSMs, the Hurst exponent can be calculated as the inverse of its fractal dimension, so algorithm FD1 can be used to calculate the Hurst exponent or the fractal dimension of any FBM or FLSM.

Our first idea is as follows. Let $\alpha: I \rightarrow \mathbb{R}$ be a parametrization of a real curve, where $I=[0,1]$. Let $\boldsymbol{\Gamma}$ be the natural fractal structure on $I$ and let also $\Delta$ be the fractal structure induced by $\boldsymbol{\Gamma}$ in $\alpha(I)$. Recall that we are going to determine the fractal dimension by means of Definition 2.

Indeed, let $s$ be a positive real number. We have that any element $A$ of each level $\Delta_{n}$ of the induced fractal structure $\boldsymbol{\Delta}$ can be written as $A=A_{1} \cup A_{2}$, where $A_{1}, A_{2} \in \Delta_{n+1}$. Suppose that the fractal structure $\boldsymbol{\Delta}$ is regular enough, so $\operatorname{diam}\left(A_{1}\right) \simeq \operatorname{diam}\left(A_{2}\right)$. Hence, if we denote by $a=\operatorname{diam}(A)$ and $b=\operatorname{diam}\left(A_{1}\right) \simeq \operatorname{diam}\left(A_{2}\right)$, then we can calculate the ratio between the diameter of elements of consecutive levels of $\boldsymbol{\Delta}$, namely, $r_{n}=\frac{b}{a} \in$ $(0,1)$. Let us suppose that there exists a common ratio $r$ (which could be taken as the mean of the list of ratios $\left\{r_{n}: n \in \mathbb{N}\right\}$ ) for any two elements of consecutive levels of $\Delta$.

Now, assume that there exists a value of $s$ such that $a^{s} \simeq 2 b^{s}=2 r^{s} a^{s}$. Then, it is easy to see that $\mathcal{H}_{n}^{s}(\alpha(I))=$ $\mathcal{H}_{n+1}^{s}(\alpha(I))$ for all $n \in \mathbb{N}$, and hence there exists $\mathcal{H}^{s}(\alpha(I))$ which is a finite quantity, so that $\operatorname{dim}_{\Delta}(\alpha)=s$. 
On the other hand, note that if $a^{s} \simeq 2 b^{s}=2 r^{s} a^{s}$, then $2 r^{s} \simeq 1$, and hence $r \simeq 2^{\frac{-1}{s}}$. Accordingly, $s \simeq \frac{-1}{\log _{2}(r)}$ would be a suitable estimation of the fractal dimension. Therefore $\operatorname{dim}_{\Delta}(\alpha) \simeq \frac{-1}{\log _{2}(r)}$.

Next, we formalize the ideas sketched above.

Theorem 1. Let $\alpha: I \rightarrow \mathbb{R}$ be a sample function of a random process $\boldsymbol{X}$ with stationary and self-affine increments with parameter $H$, where $I=[0,1]$. Let $\boldsymbol{\Gamma}$ be the natural fractal structure on $I$ and $\boldsymbol{\Delta}$ the fractal structure induced by $\boldsymbol{\Gamma}$ in $\alpha(I)$. Then $\operatorname{dim}_{\boldsymbol{\Delta}}(\alpha)=\frac{1}{H}$ and

$$
M\left(T_{n}, \omega\right) \sim 2^{H} M\left(T_{n+1}, \omega\right)
$$

with $T_{n}=\frac{1}{2^{n}}$.

Proof. Let $n \in \mathbb{N}$. Since $\mathbf{X}$ has stationary and self-affine increments with parameter $H$, by equation (4) it follows that

$$
M\left(T_{n}, \omega\right) \sim\left(\frac{1}{2^{n}}\right)^{H} M(1, \omega)
$$

and similarly,

$$
M\left(T_{n+1}, \omega\right) \sim\left(\frac{1}{2^{n+1}}\right)^{H} M(1, \omega) .
$$

From equations (7) and (8) we deduce equation (6). Now, by equation (6) we have that $M\left(T_{n}, \omega\right)^{\frac{1}{H}} \sim$ $2 M\left(T_{n+1}, \omega\right)^{\frac{1}{H}}$.

On the other hand, by $\operatorname{Remark} 1,\{\operatorname{diam}(A): A \in$ $\left.\Delta_{n}\right\}$ is a sample of the random variable $M\left(T_{n}, \omega\right)$, so $\left\{\operatorname{diam}(A)^{\frac{1}{H}}: A \in \Delta_{n}\right\}$ is a sample of the random variable $M\left(T_{n}, \omega\right)^{\frac{1}{H}}$. Since $M\left(T_{n}, \omega\right)^{\frac{1}{H}} \sim 2 M\left(T_{n+1}, \omega\right)^{\frac{1}{H}}$, then the mean of any sample of $M\left(T_{n}, \omega\right) \frac{1}{H}$ must be the double of the mean of any sample of $M\left(T_{n+1}, \omega\right)^{\frac{1}{H}}$, and hence

$$
\frac{\sum\left\{\operatorname{diam}(A)^{\frac{1}{H}}: A \in \Delta_{n}\right\}}{2^{n}}=2 \frac{\sum\left\{\operatorname{diam}(B)^{\frac{1}{H}}: B \in \Delta_{n+1}\right\}}{2^{n+1}}
$$

which implies that $\mathcal{H}_{n}^{\frac{1}{H}}(\alpha(I))=\mathcal{H}_{n+1}^{\frac{1}{H}}(\alpha(I))$. Thus, there exists $\mathcal{H}^{\frac{1}{H}}(\alpha(I)) \in(0, \infty)$ and then $\operatorname{dim}_{\Delta}(\alpha)=\frac{1}{H}$.

Theorem 1 is quite general. Next we apply it to FBMs and FLSMs.

Corollary 1. Let $\alpha: I \rightarrow \mathbb{R}$ be a sample function of a $F B M \boldsymbol{X}$ with parameter $H$, where $I=[0,1]$. Let $\boldsymbol{\Gamma}$ be the natural fractal structure on $I$ and $\boldsymbol{\Delta}$ the fractal structure induced by $\boldsymbol{\Gamma}$ in $\alpha(I)$. Then, $\operatorname{dim}_{\boldsymbol{\Delta}}(\alpha)=\frac{1}{H}$.

Proof. The increments of any FBM are stationary and self-affine with parameter $H$ by [33], Theorem 3.3, so it suffices with applying Theorem 1.

The following result provides sufficient conditions about a random process $\mathbf{X}$ in order to verify equation (3).
Theorem 2. Let $\boldsymbol{X}$ be a $H$-self similar random process with stationary increments. Then $\boldsymbol{X}$ has self-affine increments with parameter $H$.

Proof. First of all, note that $X(0, \omega)=0$ for all $\omega \in \Omega$, since $\mathbf{X}$ is a $H$-self similar process. On the other hand, the stationariness of the increments of $X$ leads to

$$
\begin{gathered}
\frac{1}{h^{H}}\left\{X\left(t_{0}+h a, \omega\right)-X\left(t_{0}, \omega\right)\right\} \sim \frac{1}{h^{H}}\{X(h a, \omega)-X(0, \omega)\} \\
\sim \frac{1}{h^{H}} X(h a, \omega) \text { for all } t_{0} \geq 0 \text { and all } h>0 .
\end{gathered}
$$

Furthermore, the next equation is also based on the $H$-self similarity of $\mathbf{X}$ :

$$
\frac{1}{h^{H}} X(h a, \omega) \sim \frac{1}{h^{H}} h^{H} X(a, \omega) \sim X(a, \omega)
$$

and now it is clear that

$$
X(a, \omega) \sim X(a, \omega)-X(0, \omega) \sim X\left(t_{0}+a, \omega\right)-X\left(t_{0}, \omega\right)
$$

for all $t_{0} \geq 0$. Thus, $\mathbf{X}$ has self-affine increments with exponent $H$.

Hence, it is possible to explore the Hurst exponent of any FLSM by means of its fractal dimension. Indeed, the proof of the next corollary is now clear from the previous results.

Corollary 2. Let $\alpha: I \rightarrow \mathbb{R}$ be a sample function of a FLSM $\boldsymbol{X}$ with parameter $H$, where $I=[0,1]$. Let $\boldsymbol{\Gamma}$ be the natural fractal structure on $I$ and $\boldsymbol{\Delta}$ the fractal structure induced by $\boldsymbol{\Gamma}$ in $\alpha(I)$. Then, $\operatorname{dim}_{\Delta}(\alpha)=\frac{1}{H}$.

Proof. Note that $\mathbf{X}$ is a $H$-self similar random process with stationary increments (see [34]). Thus, by Theorem 2, we have that $\mathbf{X}$ has self-affine increments with exponent $H$. Hence, Theorem 1 gives $\operatorname{dim}_{\Delta}(\alpha)=\frac{1}{H}$.

For practical applications the next remark is useful.

Remark 2. Note that though each fractal structure has a countable number of levels, in practical applications we are going to work with a finite number of them. That number of levels depends on the data number of the curve or the time series we are analyzing. Indeed, let $d$ be the length of the data series. Then, the maximum level that we can explore is $n \simeq \log _{2}(d)$.

Now, by Remark $1,\left\{\operatorname{diam}(A): A \in \Delta_{n}\right\}$ is a sample of the random variable $M\left(T_{n}, \omega\right)$ for all $n \in \mathbb{N}$. Let $d_{n}$ be the sample mean of $\left\{\operatorname{diam}(A): A \in \Delta_{n}\right\}$, which provides a good approximation of the mean of $M\left(T_{n}, \omega\right)$. By equation (6) of Theorem 1, it follows that $r_{n}=\frac{d_{n+1}}{d_{n}}$ must be equal to a constant $r$ and that $r=\frac{1}{2^{H}}$. It follows that $H=-\log _{2}(r)$ and by Theorem $1, \operatorname{dim}_{\Delta}(\alpha) \simeq \frac{-1}{\log _{2}(r)}$.

We can use the previous comments to give the first algorithm to calculate both the fractal dimension and the exponent $H$ of a random process $\mathbf{X}$ in the hypothesis of Theorem 1. Indeed, the procedure is as follows. 


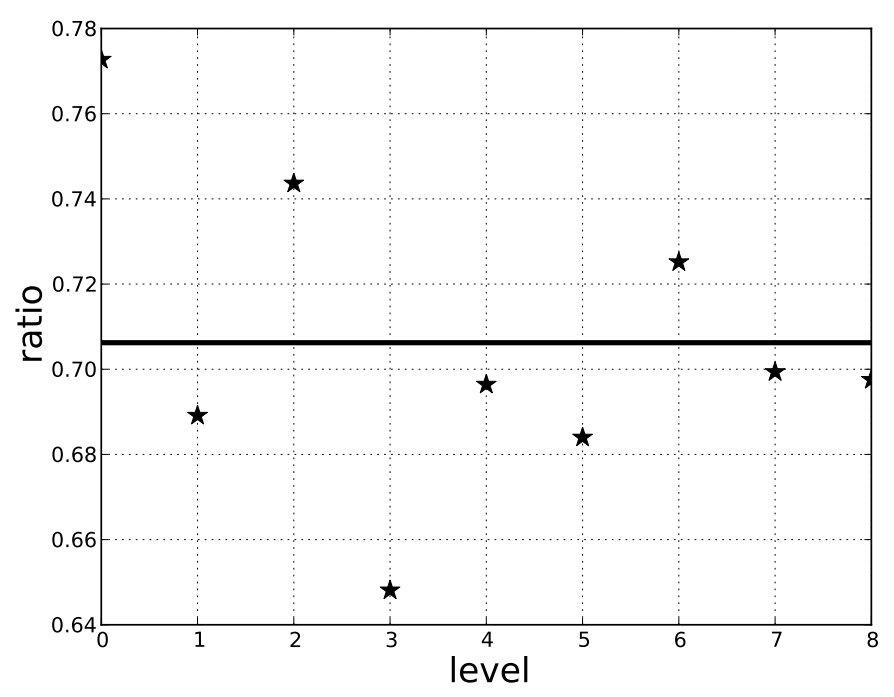

Fig. 2. Algorithm FD1 in action. Points represent the values $r_{n}$ and the line represent $r$, their mean (see the description of algorithm FD1). This was calculated for a 2048 point BM.

\section{Algorithm 1 (Algorithm FD1)}

1. Calculate $d_{n}$ as the mean of each collection $\{\operatorname{diam}(A)$ : $\left.A \in \Delta_{n}\right\}$, for $n \in\left\{1, \ldots, \log _{2}(d)\right\}$;

2. let $r_{n}=\frac{d_{n+1}}{d_{n}}$ for all $n \in\left\{1, \ldots, \log _{2}(d)-1\right\}$;

3. let $r$ be the mean of $\left\{r_{n}: n \in\left\{1, \ldots, \log _{2}(d)-1\right\}\right\}$;

4. $\operatorname{dim}_{\Delta}(\alpha)=\frac{-1}{\log _{2}(r)}$ and $H=-\log _{2}(r)$.

Figure 2 shows a graphical representation of the calculation of the fractal dimension of a time series by FD1. In this case, the coefficients $r_{n}$ are plotted as well as their mean value $r$ (the horizontal line) for a 2048 point time series (note that the induced fractal structure $\boldsymbol{\Delta}$ has 11 levels) which corresponds to a BM. Note that the quantity $r$ leads to the fractal dimension value and to the Hurst exponent of that time series.

\subsection{Two new algorithms based on statistical sth-moments: FD2 and FD3}

In this section, we introduce an alternative approach to calculate the fractal dimension of a time series which is based on statistical moments of certain random variables. Indeed, let $X$ be a random variable. Recall that its statistical $s$ th moment is defined by $m_{s}(X)=E\left(X^{s}\right)$ for each $s>0$ (if that expected value exists). Furthermore, let $\left\{x_{k}: k=1, \ldots, n\right\}$ be a sample of length $n$ of the random variable $X$. Thus, its sample $s$ th-moment is calculated as

$$
m_{s}(X)=\frac{\sum_{i=1}^{n} x_{i}^{s}}{n} .
$$

Next, we present our main theorem which allows us to calculate the fractal dimension for a wide range of curves.

Theorem 3. Let $\alpha: I \rightarrow \mathbb{R}$ be a sample function of a random process $\boldsymbol{X}$, with $I=[0,1]$. Let $\boldsymbol{\Gamma}$ be the natural fractal structure on $I$ and $\boldsymbol{\Delta}$ the fractal structure induced by $\boldsymbol{\Gamma}$ in $\alpha(I)$. Let $X_{n}=M\left(\frac{1}{2^{n}}, \omega\right)$ be the random variable which provides the statistical distribution of the list of diameters $\left\{\operatorname{diam}(A): A \in \Delta_{n}\right\}$, for all $n \in \mathbb{N}$. Suppose also that there exists a positive real number $s$ that verifies the next two conditions:

1. there exists $m_{s}\left(X_{n}\right)$ for all $n \in \mathbb{N}$;

2. $m_{s}\left(X_{n}\right)=2 m_{s}\left(X_{n+1}\right)$ for all $n \in \mathbb{N}$.

Then, $\operatorname{dim}_{\Delta}(\alpha)=s$.

Proof. The sample sth-moment of $\left\{\operatorname{diam}(A): A \in \Delta_{n}\right\}$ is approximately equal to $m_{s}\left(X_{n}\right)$, that is,

$$
m_{s}\left(X_{n}\right)=\frac{\sum\left\{\operatorname{diam}(A)^{s}: A \in \Delta_{n}\right\}}{2^{n}}
$$

since each level $\Delta_{n}$ of the induced fractal structure $\boldsymbol{\Delta}$ has $2^{n}$ elements. Hence,

$$
\mathcal{H}_{n}^{s}(\alpha(I))=\sum\left\{\operatorname{diam}(A)^{s}: A \in \Delta_{n}\right\}=2^{n} m_{s}\left(X_{n}\right)
$$

and a similar argument leads to

$$
\mathcal{H}_{n+1}^{s}(\alpha(I))=2^{n+1} m_{s}\left(X_{n+1}\right) .
$$

Equations (9) and (10) and hypothesis (2) of the theorem allow us to affirm that $\mathcal{H}_{n}^{s}(\alpha(I))=\mathcal{H}_{n+1}^{s}(\alpha(I))$ for all natural number $n$, which implies that $\mathcal{H}^{s}(\alpha(I))$ is a finite and positive real number. Therefore, $\operatorname{dim}_{\Delta}(\alpha)=s$.

Remark 3. Note that the main theoretical hypothesis in Theorem 3, namely, $m_{s}\left(X_{n}\right)=2 m_{s}\left(X_{n+1}\right)$ for all $n \in \mathbb{N}$, is usually verified in most practical applications. This fact is shown empirically in Section 6.

Now, we use the key Theorem 3 to give two new algorithms to calculate the fractal dimension and the Hurst exponent of any time series.

As before, recall that any fractal structure has a countable number of levels. Nevertheless, in practical applications, the quantity of levels we are going to work with depends on the data number (see Rem. 2). In order to determine the number of levels of the fractal structure $\boldsymbol{\Delta}$, note that if $d$ is the length of the data, then $\log _{2}(d)$ is the deeper level we can consider.

The key condition we are going to check in our algorithms is about the main theoretical hypothesis in Theorem 3, namely,

$$
m_{s}\left(X_{n}\right)=2 m_{s}\left(X_{n+1}\right)
$$

where the random variable $X_{n}$ provides the statistical distribution of the list of diameters $\left\{\operatorname{diam}(A): A \in \Delta_{n}\right\}$, for all $n \in \mathbb{N}$. Equivalently, it suffices with checking the condition $\frac{m_{s}\left(X_{k}\right)}{m_{s}\left(X_{k+1}\right)}=2$ for $k \in\left\{1,2, \ldots, \log _{2}(d)-1\right\}$. Indeed, we introduce the next algorithm to estimate the fractal dimension. 


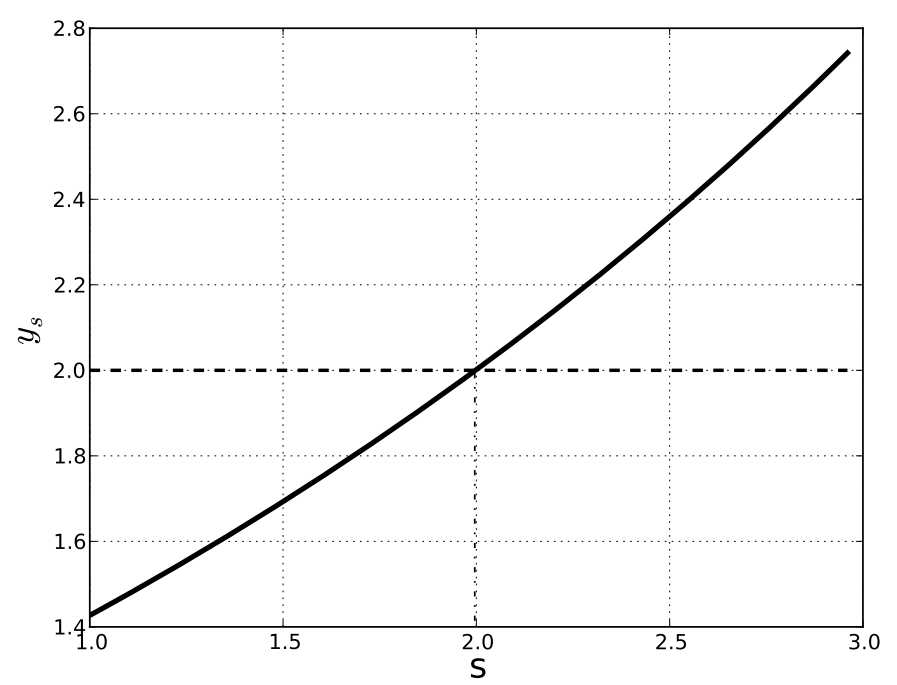

Fig. 3. Algorithm FD2 in action. Example of the graph representation of $\overline{y_{s}}$ vs. $s$ for a 2048 length time series of a BM (see the description of algorithm FD2). The fractal dimension is close to $2(H=0.5)$ and corresponds with the value of $s$ which makes $\overline{y_{s}}=2$.

\section{Algorithm 2 (Algorithm FD2)}

1. For each $s>0$, compute the list $y_{s}=\left\{y_{k, s}: k \in\right.$ $\left.\left\{1,2, \ldots, \log _{2}(d)-1\right\}\right\}$, where $y_{k, s}=\frac{m_{s}\left(X_{k}\right)}{m_{s}\left(X_{k+1}\right)} ;$

2. let $\overline{y_{s}}$ be the mean of each list $y_{s}$;

3 . find $s_{0}$ such that $\overline{y_{s_{0}}}=2$ (note that $\left\{\left(s, \overline{y_{s}}\right): s>0\right\}$ is a $s$-increasing function);

4. $\operatorname{dim}_{\Delta}(\alpha)=s_{0}$ (Thm. 3) and $H=\frac{1}{s_{0}}$ (Thm. 1).

Figure 3 gives an illustration of the calculations in algorithm FD2. It represents the graph of $\overline{y_{s}}$ in function of $s$. Note that $\overline{y_{s}}$ is an increasing funcion of $s$, so it is easy to find the value $s_{0}$ of $s$ which makes $\overline{y_{s_{0}}}=2$. It is the value of the fractal dimension. In this case, a 2048 point time series which corresponds to a BM is used.

Next we introduce algorithm FD3, which is an alternative to FD2 and is based on the same Theorem 3.

First, note that equation (11) is equivalent to

$$
m_{s}\left(X_{n}\right)=\frac{1}{2^{n-1}} m_{s}\left(X_{1}\right)
$$

for all $n \in \mathbb{N}$. Thus, if we take 2-base logarithms in equation (12), then

$$
\log _{2}\left(m_{s}\left(X_{n}\right)\right)=-n+\gamma
$$

where $\gamma=1+\log _{2}\left(m_{s}\left(X_{1}\right)\right)$ remains constant. This gives a linear relation between $\log _{2}\left(m_{s}\left(X_{n}\right)\right)$ and $n$ that can be used to calculate the fractal dimension.

Next, we provide the technical description of the algorithm based on the above ideas.

\section{Algorithm 3 (Algorithm FD3)}

1. For each $s>0$, consider the 2D-point cloud $\left\{\left(k, \beta_{k, s}\right)\right.$ : $\left.k \in\left\{1, \ldots, \log _{2}(d)\right\}\right\}$ with $\beta_{k, s}=\log _{2}\left(m_{s}\left(X_{k}\right)\right)$, and let $\beta_{s}$ be the slope of the regression line of the cloud;
2. consider the increasing function $\left\{\left(s, \beta_{s}\right): s>0\right\}$, and determine $s_{1}$ such that $\beta_{s_{1}}=-1$;

3. $\operatorname{dim}_{\Delta}(\alpha)=s_{1}$ (Thm. 3 and Eq. (13)) and $H=\frac{1}{s_{1}}$ (Thm. 1).

Algorithm FD3 can be used to check the main hypothesis in Theorem 3, namely, $m_{s}\left(X_{n}\right)=2 m_{s}\left(X_{n+1}\right)$ for all $n \in \mathbb{N}$. Indeed, note that equation (13) is equivalent to condition (2) in Theorem 3, so to check that the empirical data verify that condition, we only have to check that equation (13) is verified, that is, the regression coefficient of equation (13) is close to (1).

A graphical interpretation similar to that one given in Figure 3 could be performed.

\section{Testing the accuracy of FD methods}

We explore the accuracy of FD methods developed in the previous section. These new approaches to estimate the Hurst exponent are specially appropriated when analyzing short length time series. Note that it becomes a significative advantage compared to other algorithms, like the classical R/S analysis which does not work fine for short time series.

The accuracy of FD1 has been tested before applying it to capital markets. Indeed, we have used Monte Carlo simulation as follows: we have generated 10000 BMs (Hurst exponent $H=0.5$ ) with a length of 1024 and have calculated the Hurst exponent considering that prices change about 128 times per day (actually, they usually change much more, but for simulation purposes, 128 times is enough). This is done so that we can simulate the maximum and minimum of each day. The mean of the Hurst exponent was found to be 0.50 and the standard deviation 0.04 which is very close to 0 . Thus, this means that FD1 works appropriately as a self-similarity estimator because the computed value for the Hurst exponent is near to 0.5 in the case of BMs. A similar analysis to the previous one was carried out for $H=0.25$ (that is, anti-persistent FBMs) and for $H=0.75$. In the first case, the mean was found to be 0.33 and the standard deviation was equal to 0.04 . On the other hand, for $H=0.75$, the mean of the Hurst exponent was 0.74 with a standard deviation of 0.07 . We can see that for small values of $H$, FD1 lost accuracy, while it is quite accurate for values of $H$ near to or greater than 0.5 (see also Fig. 5). Remember that, by Section 4.2 , FD1 is correct asymptotically.

For all these values of $H$, namely, $H \in\{0.25,0.5,0.75\}$, analogous analysis also were carried out for time series with lengths of 32 and 256. Table 1 contains the complete results.

On the other hand, a similar analysis to the previous one was carried out for algorithms FD2 and FD3. Indeed, for both algorithms we have tested its accuracy via the Monte Carlo method by generating 10000 FBMs for different values of $\mathrm{H}$. The length of all the time series was equal to 1024 and we also consider that prices change about 128 times per day. Firstly, we tested the behavior of FD2 for BMs. In this case, the mean of the estimated 
Table 1. Influence of the length of the series in the Hurst exponent using different approaches. The results have been obtained by Monte-Carlo simulation of 10000 FBMs with Hurst exponents $H \in\{0.25,0.5,0.75\}$ and time series of lengths equal to 32,256 and 1024. The data displayed in bold corresponds with the best computed mean in each case.

\begin{tabular}{|c|c|c|c|c|c|c|}
\hline & & & \multicolumn{2}{|l|}{$\mathrm{R} / \mathrm{S}$} & \multicolumn{2}{|c|}{$\overline{D F A}$} \\
\hline $\bar{H}$ & \multicolumn{2}{|c|}{ Length } & Mean & Std & Mean & Std \\
\hline \multirow[t]{3}{*}{0.25} & \multicolumn{2}{|c|}{32} & $0.60 \quad 0$ & 0.07 & -0.00 & 0.37 \\
\hline & \multicolumn{2}{|c|}{256} & $0.41 \quad 0$ & 0.06 & 0.11 & .09 \\
\hline & \multicolumn{2}{|c|}{1024} & 0.34 & 0.05 & 0.16 & .06 \\
\hline \multirow[t]{3}{*}{0.5} & \multicolumn{2}{|c|}{32} & 0.66 & 0.07 & 0.46 & 0.43 \\
\hline & \multicolumn{2}{|c|}{256} & 0.57 & 0.08 & 0.47 & .10 \\
\hline & \multicolumn{2}{|c|}{1024} & $0.54 \quad 0$ & 0.08 & 0.48 & .06 \\
\hline \multirow[t]{3}{*}{0.75} & \multicolumn{2}{|c|}{32} & 0.69 & 0.07 & 0.68 & .42 \\
\hline & \multirow{2}{*}{\multicolumn{2}{|c|}{$\begin{array}{c}256 \\
1024\end{array}$}} & 0.71 & 0.10 & 0.71 & .12 \\
\hline & & & 0.74 & 0.11 & $0.72 \quad 0$ & .08 \\
\hline & \multirow{2}{*}{\multicolumn{2}{|c|}{ Length }} & \multicolumn{2}{|l|}{ GM1 } & \multicolumn{2}{|c|}{ GM2 } \\
\hline$H$ & & & Mean & Std & Mean & Std \\
\hline \multirow[t]{3}{*}{0.25} & \multicolumn{2}{|c|}{32} & 0.30 & 0.31 & $0.31 \quad 0$ & .06 \\
\hline & \multicolumn{2}{|c|}{256} & 0.28 & 0.11 & 0.30 & .03 \\
\hline & \multicolumn{2}{|c|}{1024} & 0.28 & 0.07 & 0.29 & .02 \\
\hline \multirow[t]{3}{*}{0.5} & \multicolumn{2}{|c|}{32} & $0.66 \quad 0$ & 0.30 & 0.51 & .08 \\
\hline & \multicolumn{2}{|c|}{256} & $0.57 \quad 0$ & .11 & 0.51 & .03 \\
\hline & \multicolumn{2}{|c|}{1024} & 0.54 & .07 & 0.51 & .04 \\
\hline \multirow[t]{3}{*}{0.75} & \multicolumn{2}{|c|}{32} & 0.98 & .27 & $\begin{array}{ll}0.73 \\
\end{array}$ & .11 \\
\hline & \multicolumn{2}{|c|}{256} & $0.86 \quad 0$ & .11 & 0.74 & .07 \\
\hline & \multicolumn{2}{|c|}{1024} & $0.82 \quad 0$ & .08 & 0.74 & .05 \\
\hline & $\overline{F D}$ & & & $\mathrm{D} 2$ & & 3 \\
\hline$H$ & Mean & Std & Mean & Std & Mean & Std \\
\hline 0.25 & 0.37 & 0.09 & 0.25 & 0.08 & 0.24 & 0.09 \\
\hline & 0.34 & 0.05 & 0.26 & 0.05 & 0.25 & 0.05 \\
\hline & 0.33 & 0.04 & 0.26 & 0.04 & 0.25 & 0.04 \\
\hline 0.5 & 0.50 & 0.09 & 0.49 & 0.10 & 0.48 & 0.10 \\
\hline & 0.50 & 0.05 & 0.50 & 0.06 & 0.49 & 0.06 \\
\hline & 0.50 & 0.04 & 0.50 & 0.05 & 0.49 & 0.04 \\
\hline 0.75 & 0.72 & 0.13 & 0.71 & 0.15 & 0.69 & 0.15 \\
\hline & 0.73 & 0.08 & 0.73 & 0.09 & 0.72 & 0.10 \\
\hline & 0.74 & 0.07 & 0.73 & 0.07 & 0.73 & 0.07 \\
\hline
\end{tabular}

Hurst exponent was found to be 0.50 with a standard deviation of 0.05 . Thus, FD2 works appropriately in the case of random walks. Furthermore, for $H=0.25$, the mean of the Hurst exponent was found to be 0.26 with a standard deviation of 0.04 , and for $H=0.75$, the mean was found to be 0.73 with a standard deviation equal to 0.07 .

Similarly, algorithm FD3 was tested before being applied to capital markets. Thus, for $H=0.5$ we found that the mean was equal to 0.49 with a standard deviation of 0.04 . Since the mean is very close to 0.5 and the deviation is quite small, we conclude that FD3 works fine for analyzing BMs. Moreover, note that the results were also interesting for anti-persistent FBMs $(H=0.25)$, since the mean was 0.25 with a slight standard deviation, 0.04 . The results for $H=0.75$ were as follows: the mean was found to be 0.73 with a standard deviation equal to 0.07 . Table 1 contains all the data generated in the analysis.
As can be seen in Table 1 as well as in Figures 4 and 5, both FD2 and FD3 algorithms work fine for all range of $\mathrm{H}$.

Figure 5 shows a graphical comparison among different algorithms to calculate the Hurst exponent: the fractal methods (FD1, FD2 and FD3) vs. geometrical algorithms (GM1 and GM2) vs. the classical procedures (R/S analysis and DFA). The graphs were plotted for a Monte Carlo simulation of $1000 \mathrm{FBMs}$ for different Hurst exponents $H \in(0,1)$ with a length of 256 (with 128 changes per day). Note that both FD2 and FD3 result versatile in order to estimate all the possible values of $H$ : persistent, anti-persistent and random ones. Nevertheless, for Hurst exponents less than or equal to 0.3 approximately, FD1 becomes less accurate than the other FD methods. Moreover, note that GM2 is not as accurate as both FD2 and FD3 in order to estimate small values of the Hurst exponent. On the other hand, algorithm GM1 does not provide estimations so accurate as fractal ones. Note that the classical algorithms have been improved by both fractal and geometrical techniques.

We also like to point out the influence of the length of the time series when estimating the Hurst exponent. Indeed, one of the advantages that our fractal dimension techniques have is about its accuracy when analyzing short length time series. Unlike R/S analysis, note that the accuracy of FD methods is not too influenced by the length of the time series, in the sense that we obtain means close to its theoretical values with slight standard deviations when taking short time series. This fact leads to a significant advantage of the fractal approaches as well as it happens with the geometrical estimations. In this way, Table 2 compares the influence of the length of the series using fractal, geometrical and classical procedures. The experiments were carried out for a Monte Carlo simulation of 1000 BMs (Hurst exponent equal to 0.5), and with 128 changes per day. Note that for short length time series, both FD1 and FD2 methods returns the expected mean 0.5 with slight standard deviations. In this way, FD3 algorithm also provides interesting results. On the other hand, note that R/S analysis needs long time series (with lengths from 4096) in order to provide means near to 0.5 . For lower lengths than 512, the obtained standard deviations using DFA are greater than those obtained by fractal techniques. Moreover, GM2 is the algorithm that have lower standard deviations for all lengths.

An interesting graphical comparison of the accuracy of the different approaches to estimate the Hurst exponent was carried out in Figure 6 for $H=0.5$. The first picture compares the histogram of the estimated $H$ using the classical approaches (R/S analysis and DFA) vs. the geometrical algorithms (GM1 and GM2). The highest mode histogram corresponds to algorithm GM2, whose mean is close to 0.5 and presents the slightest deviation. On the other hand, the image at the bottom contains the histograms of the estimated $H$ using algorithms FD compared with DFA (the lowest mode histogram). Note that all algorithms FD provide similar and accurate estimations of the Hurst exponent, and the histogram is more centered at 0.5 than DFA. The analysis were carried out 


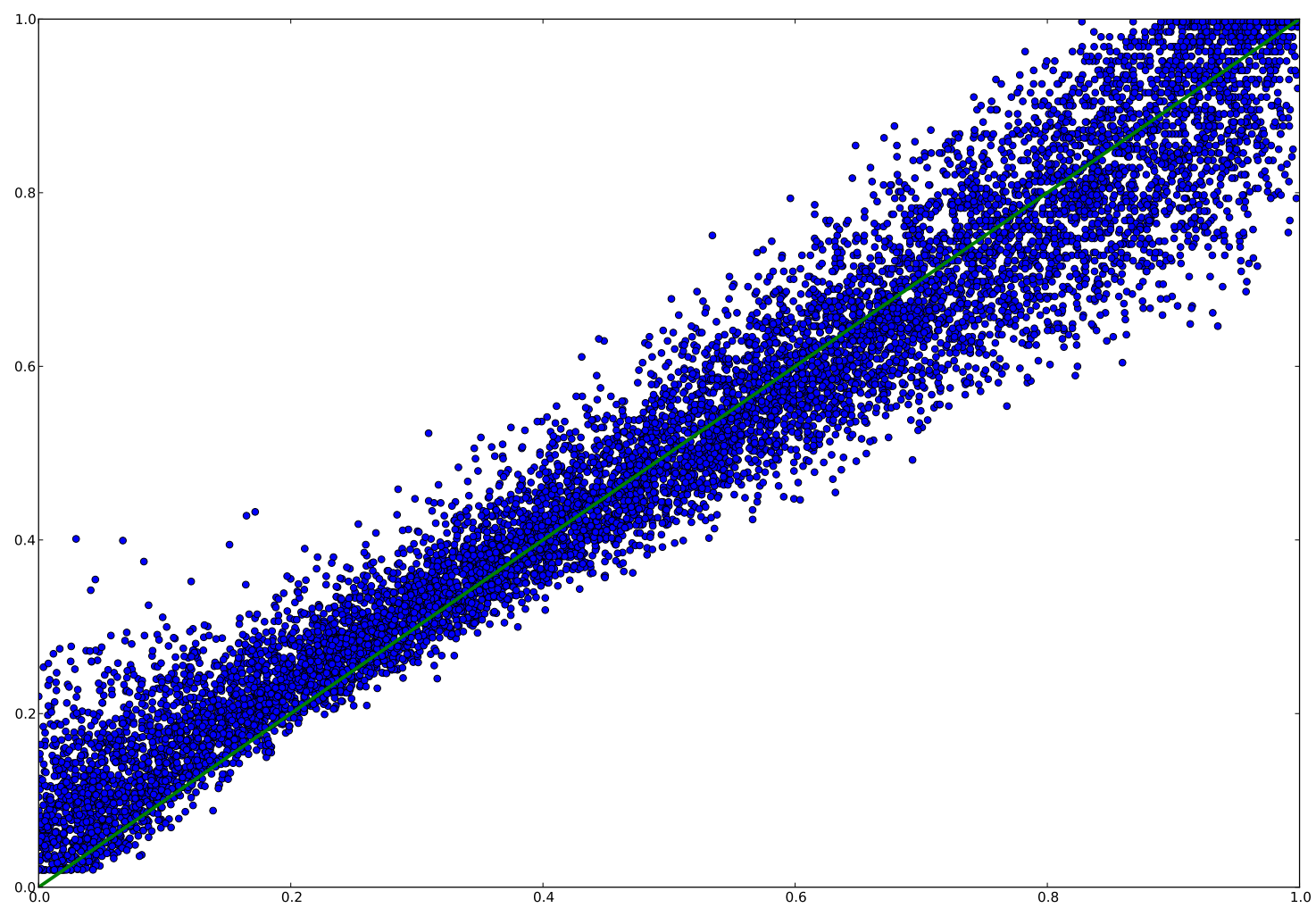

Fig. 4. (Color online) Accuracy of algorithm FD2 when estimating $H$. The graph representation compares the theoretical Hurst exponent vs. the empirical one given by FD2. The experiments were carried out with a Monte Carlo simulation of 10000 FBMs with a uniform random Hurst exponent between 0 and 1 and with lengths of 1024 (with 128 changes per day). Note that each point of the cloud corresponds with one simulation.

by Monte Carlo simulation of 10000 Brownian motions with a length of 1024 and 128 changes per day.

\section{Empirical applications}

Two empirical applications of our fractal dimension techniques are shown in this section. In the first of them, the Hurst exponent of several international stock market indexes is estimated. In the second one, we use algorithm FD2 to calculate the Hurst exponent of individual stocks.

\subsection{Exploring long memory in stock market indexes}

We analyze market memory in several stock indexes through the Hurst exponent. In this way, the Hurst exponent of the following international indexes is estimated by means of FD methods: Cac40, FTSE100, NIKKEI225, Ibex35, DAX, S\&P500, Nasdaq100 and Nasdaq composite.

Table 3 contains the Hurst exponent estimations obtained by algorithms FD1, FD2 and FD3. In this way, note that the length of each time series was chosen to be equal for all the studied market indexes. Indeed, time series of 256 (one year) and 1024 (4 years) data were considered in order to estimate $H$. Thus, since the length of all the time series was the same, a possible comparison of the results that the algorithms provided could be done in the same terms. The last day of the data used in both cases was April 11, 2011.

With a confidence level of $95 \%$, we have calculated the empirical confidence intervals of each algorithm for the Hurst exponent of a BM of length 256: $(0.408,0.602)$ for FD1, $(0.395,0.620)$ for FD2 and $(0.384,0.609)$ for FD3. On the other hand, for BMs of length 1024, the confidence intervals were found to be $(0.430,0.580),(0.416,0.594)$ and $(0.412,0.581)$ for FD1, FD2 and FD3, respectively, for a confidence level of $95 \%$.

Note that the estimation of the Hurst exponent for 1024 length time series of S\&P500, Nasdaq100 and Nasdaq composite provides a value very close to the upper limit of the corresponding confidence interval (for algorithm FD1). Moreover, the Hurst exponent estimations for both 256 and 1024 length time series are near to the upper limit of the confidence intervals in the case of NIKKEI225 index. In particular, for 1024 length time series, the Hurst exponent calculated using FD1 equals to the upper limit of the corresponding confidence interval. Also, the FD2 and FD3 Hurst exponent estimations for 1024 length time series of NIKKEI225 are close to the upper limit of their intervals. Anyway, all estimations of $H$ lie within the confidence intervals. Therefore, we can say that the behavior of the evolution of these international stock market indexes is random: we cannot conclude that their Hurst exponent values are statistically different from 0.5 in any case. 

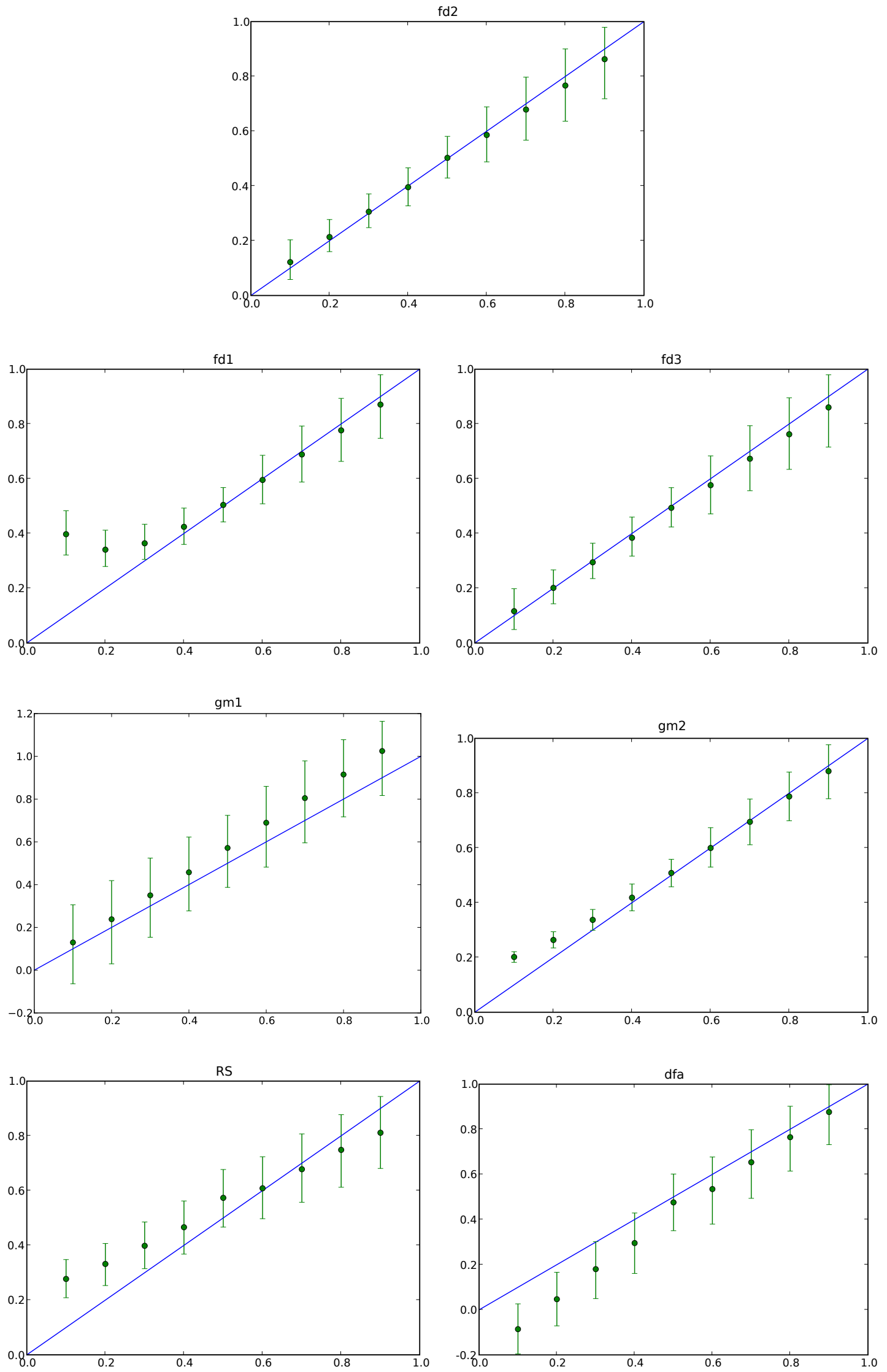

Fig. 5. (Color online) Accuracy of different approaches to estimate the Hurst exponent. Horizontal bars represent the confidence intervals of the empirical distribution of the algorithms at a confidence level of $95 \%$. 
Table 2. Influence of the length of the series in the Hurst exponent estimation using different approaches. The analysis were performed by Monte Carlo simulation of $10000 \mathrm{BMs}$ $(H=0.5)$. In each case, the Hurst exponents displayed in bold corresponds to the best computed mean of $H$.

\begin{tabular}{lcccc}
\hline & \multicolumn{2}{c}{ R/S } & \multicolumn{2}{c}{ DFA } \\
\hline Length & Mean & Std & Mean & Std \\
\hline $\mathbf{3 2}$ & 0.66 & 0.07 & 0.46 & 0.43 \\
$\mathbf{6 4}$ & 0.69 & 0.05 & 0.45 & 0.22 \\
$\mathbf{1 2 8}$ & 0.60 & 0.08 & 0.47 & 0.14 \\
$\mathbf{2 5 6}$ & 0.57 & 0.08 & 0.47 & 0.10 \\
$\mathbf{5 1 2}$ & 0.55 & 0.08 & 0.48 & 0.08 \\
$\mathbf{1 0 2 4}$ & 0.54 & 0.08 & 0.48 & 0.06 \\
$\mathbf{2 0 4 8}$ & 0.54 & 0.06 & 0.49 & 0.05 \\
$\mathbf{4 0 9 6}$ & 0.53 & 0.06 & 0.49 & 0.04 \\
$\mathbf{8 1 9 2}$ & 0.52 & 0.04 & 0.49 & 0.04 \\
\hline
\end{tabular}

\begin{tabular}{lcccc}
\hline & \multicolumn{2}{c}{ GM1 } & \multicolumn{2}{c}{ GM2 } \\
\hline Length & Mean & Std & Mean & Std \\
\hline $\mathbf{3 2}$ & 0.66 & 0.30 & 0.51 & 0.08 \\
$\mathbf{6 4}$ & 0.61 & 0.20 & 0.51 & 0.06 \\
$\mathbf{1 2 8}$ & 0.59 & 0.15 & 0.51 & 0.05 \\
$\mathbf{2 5 6}$ & 0.56 & 0.11 & 0.51 & 0.04 \\
$\mathbf{5 1 2}$ & 0.55 & 0.09 & 0.51 & 0.03 \\
$\mathbf{1 0 2 4}$ & 0.55 & 0.07 & 0.51 & 0.03 \\
$\mathbf{2 0 4 8}$ & 0.54 & 0.06 & 0.51 & 0.02 \\
$\mathbf{4 0 9 6}$ & 0.53 & 0.05 & 0.51 & 0.02 \\
$\mathbf{8 1 9 2}$ & 0.53 & 0.05 & 0.51 & 0.02 \\
\hline
\end{tabular}

\begin{tabular}{llccccc}
\hline & \multicolumn{2}{c}{ FD1 } & \multicolumn{2}{c}{ FD2 } & \multicolumn{2}{c}{ FD3 } \\
\hline Length & Mean & Std & Mean & Std & Mean & Std \\
\hline $\mathbf{3 2}$ & $\mathbf{0 . 5 0}$ & 0.09 & 0.49 & 0.10 & 0.48 & 0.10 \\
$\mathbf{6 4}$ & $\mathbf{0 . 5 0}$ & 0.07 & 0.49 & 0.08 & 0.48 & 0.09 \\
$\mathbf{1 2 8}$ & $\mathbf{0 . 5 0}$ & 0.06 & 0.50 & 0.07 & 0.49 & 0.07 \\
$\mathbf{2 5 6}$ & $\mathbf{0 . 5 0}$ & 0.05 & 0.50 & 0.06 & 0.49 & 0.06 \\
$\mathbf{5 1 2}$ & $\mathbf{0 . 5 0}$ & 0.04 & 0.50 & 0.05 & 0.49 & 0.05 \\
$\mathbf{1 0 2 4}$ & $\mathbf{0 . 5 0}$ & 0.04 & 0.50 & 0.05 & 0.49 & 0.04 \\
$\mathbf{2 0 4 8}$ & $\mathbf{0 . 5 0}$ & 0.04 & 0.50 & 0.04 & 0.49 & 0.04 \\
$\mathbf{4 0 9 6}$ & $\mathbf{0 . 5 0}$ & 0.03 & 0.49 & 0.04 & 0.49 & 0.03 \\
$\mathbf{8 1 9 2}$ & $\mathbf{0 . 5 0}$ & 0.04 & 0.49 & 0.03 & 0.49 & 0.03 \\
\hline
\end{tabular}

Table 3. Hurst exponent estimations for different stock market indexes using different approaches, where 256 (one year) and 1024 (4 years) diary data where used. The Hurst exponents displayed in bold correspond to those estimations of $H$ which are close to the upper limit of the corresponding confidence interval. All estimations of $H$ lie within the confidence intervals.

\begin{tabular}{lcccccc}
\hline Index & \multicolumn{2}{c}{ FD1 } & \multicolumn{2}{c}{ FD2 } & \multicolumn{2}{c}{ FD3 } \\
\hline Last Data & $\mathbf{2 5 6}$ & $\mathbf{1 0 2 4}$ & $\mathbf{2 5 6}$ & $\mathbf{1 0 2 4}$ & $\mathbf{2 5 6}$ & $\mathbf{1 0 2 4}$ \\
Cac40 & 0.45 & 0.55 & 0.43 & 0.54 & 0.42 & 0.53 \\
FTSE100 & 0.45 & 0.53 & 0.48 & 0.48 & 0.46 & 0.49 \\
NIKKEI225 & $\mathbf{0 . 5 9}$ & $\mathbf{0 . 5 8}$ & 0.51 & $\mathbf{0 . 5 6}$ & 0.52 & $\mathbf{0 . 5 6}$ \\
Ibex35 & 0.46 & 0.54 & 0.42 & 0.51 & 0.42 & 0.52 \\
DAX & 0.48 & 0.55 & 0.53 & 0.51 & 0.49 & 0.51 \\
S\&P500 & 0.52 & $\mathbf{0 . 5 7}$ & 0.53 & 0.53 & 0.50 & 0.54 \\
Nasdaq100 & 0.52 & $\mathbf{0 . 5 7}$ & 0.56 & 0.52 & 0.51 & 0.53 \\
Nasdaq C. & 0.53 & $\mathbf{0 . 5 8}$ & 0.54 & 0.52 & 0.51 & 0.53 \\
\hline
\end{tabular}
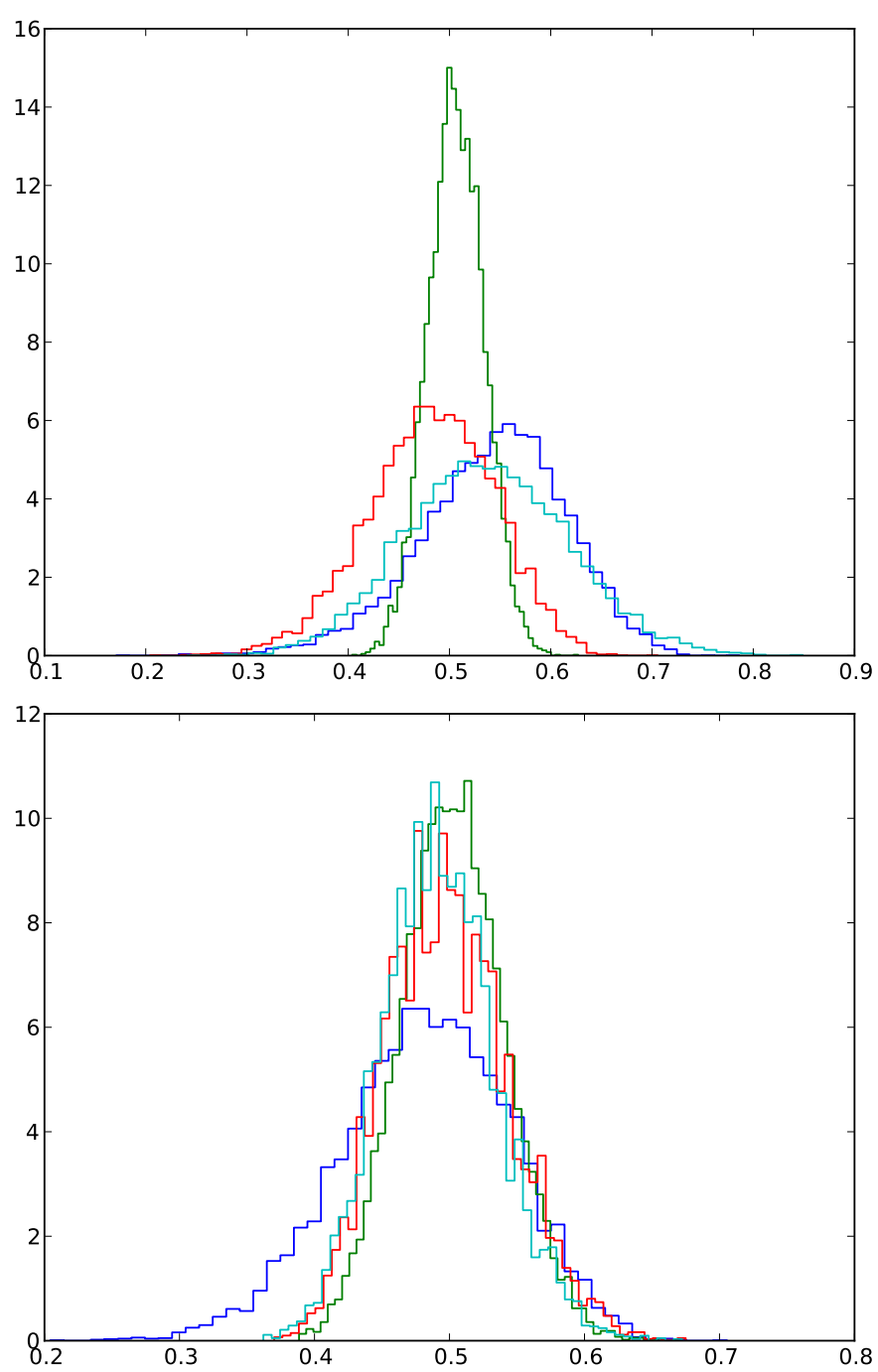

Fig. 6. (Color online) Comparison of the accuracy of different approaches to estimate the Hurst exponent. The first image contains the histogram of the estimated Hurst exponent of BMs $(H=0.5)$ of both classical and geometrical approaches while the second one compares the FD methods vs. algorithm DFA. The analysis were carried out by Monte Carlo simulation of 10000 BMs with a length of 1024 and 128 changes per day.

\subsection{The Hurst exponent for individual stocks}

In this section, we study the Hurst exponent given by FD2 for the components of the S\&P500 and the Nasdaq100 stock indexes. We make the test for different time intervals from 256 data (one year) to 4096 data (16 years). The last day of the data is April 11, 2011.

First, we check that the stocks follow the hypothesis of Theorem 3. To do that, we calculate the regression coefficient for equation (13) (see Sect. 4.3). Note that a regression coefficient close to 1 means that the linear relation is true, and hence the hypothesis of Theorem 3 is satisfied.

Following that procedure we find that in most of the cases the regression coefficient is greater than 0.99 and only for a few cases it is between 0.98 and 0.99 . Only in two cases the regression coefficient is less than 0.98 , with values 
Table 4. Percentage of stocks in the index with a Hurst exponent, given by FD2, inside the confidence intervals, at a confidence level of $95 \%$.

\begin{tabular}{lcc}
\hline Data Length & S\&P500 & Nasdaq100 \\
\hline $\mathbf{2 5 6}$ & $95 \%$ & $95 \%$ \\
$\mathbf{5 1 2}$ & $96 \%$ & $92 \%$ \\
$\mathbf{1 0 2 4}$ & $93 \%$ & $96 \%$ \\
$\mathbf{4 0 9 6}$ & $98 \%$ & $98 \%$ \\
\hline
\end{tabular}

of 0.977 and 0.979 . This means that we have empirical evidence that stocks verify the hypothesis of Theorem 3.

Second, we calculate the confidence intervals for each of the lengths. This is done by Monte Carlo simulation, calculating the Hurst exponent given by FD2 of $10000 \mathrm{BMs}$, and then calculating the inferior and superior limits of the interval so that $95 \%$ of the Hurst exponent obtained lies between them.

In Table 4 we can see the percentage of stocks for each index that have a Hurst exponent, given by FD2, inside the confidence intervals, at a confidence level of $95 \%$. From the results obtained, we cannot conclude that the stocks does not follow a BM, so there is no evidence of memory in the series.

\section{Conclusions}

To conclude, we will like to point out that this paper presents novel theoretical and empirical results in order to estimate the Hurst exponent. The concept of a fractal structure of a curve is first applied in order to provide a new definition of fractal dimension for a parametrization of a curve. Thus, we can explore long memory in time series by using fractal techniques.

In particular, we prove that for random processes with stationary and self-affine increments with parameter $H$ (which include FBMs and FLSMs with parameter $H$ ), the fractal dimension is equal to the inverse of $H$. Thus, the fractal dimension can be considered a generalization of the Hurst exponent, since the former could be computed for any real curve (not necessarily continuous). For instance, note that unlike R/S analysis and DFA (see [23]), the FD methods introduced here are able to estimate the self-similarity index of FLSMs and any other random process with stationary and self-affine increments.

We also prove some general results that are the base for three new algorithms (called FD methods) to calculate both the fractal dimension and the exponent $H$. They are based on two mathematical techniques: algorithm FD1 is based on the ratio of the diameter of elements of consecutive levels of the fractal structure, while algorithms FD2 and FD3 are based on statistical moments. All three algorithms are supported by theoretical results, which guarantees that they are correct in an asymptotic way.

On the other hand, it is well known and, in any case, easy to proof (see Tab. 1) that, for short length time series, classical methods are not accurate. Some of the solutions to avoid it that have appeared in the literature are unnecessarily artificial or complex. Algorithms FD are a simpler and more efficient solution to this problem, since they work fine with short time series. In particular, they are suitable to study financial series, where it is usual to have some extra information, like the maximum and the minimum of the day (or period). The use of this information make the algorithms FD1, FD2, FD3 and GM2 much more accurate than the classical ones.

To illustrate it, the influence of the length of the series is analyzed for all fractal, geometrical and classical algorithms to estimate $H$. As a result of this analysis, we conclude that both the fractal and the geometrical approaches perform much better than the classical methods, specially for short time series.

Finally, we have studied stock indexes and individual stocks and we find no evidence of memory using the three algorithms introduced in the paper.

We would like to thank Prof. B. McKelvey (UCLA Anderson School of Management) for his valuable comments and suggestions. On the other hand, the first author acknowledges the support of the Spanish Ministry of Science and Innovation, Grant MTM2009-12872-C02-01. The second one appreciates a FPU grant of the Spanish Ministry of Education. The third author acknowledges the support of Junta de Andalucia, Grant P09-SEJ-5404.

\section{References}

1. A.W. Lo, Econometrica 59, 1279 (1991)

2. A.W. Lo, A.C. MacKinlay, Long-term memory in stock market prices, in A Non-Random Walk Down Wall Street (Princeton University Press, 1999), Chap. 6

3. M. Greene, B. Fielitz, J. Financ. Econ. 4, 339 (1977)

4. J. Hampton, Rescaled range analysis: Approaches for the financial practitioners, Part 3, Neuro Vest Journal 27 (1996)

5. F. Lillo, J.D. Farmer, Studies in Nonlinear Dynamics and Econometrics 8, 1 (2004)

6. E. Panas, Appl. Financ. Econ. 11, 395 (2001)

7. E. Peters, Financ. Anal. J. 48, 32 (1992)

8. B.B. Mandelbrot, J.R. Wallis, Water Resourc. Res. 5, 967 (1969)

9. C.M. Jensen, J. Financ. Econ. 6, 95 (1978)

10. B.G. Malkiel, J. Econ. Perspect. 17, 59 (2003)

11. W. Goetsmann, Journal of Business 66, 249 (1993)

12. E. Peters, Chaos and Order in the Capital Markets: A New View of Cycles, Prices and Market Volatility (John Wiley \& Sons Inc., New York, 1996)

13. R. Weron, Physica A 312, 285 (2002)

14. M. Ausloos, Physica A 285, 48 (2000)

15. T. Di Matteo, T. Aste, M. Dacorogna, J. Bank. Financ. 29, 827 (2005)

16. J.W. Kantelhardt, E. Koscielny-Bunde, H.H.A. Rego, S. Havlin, A. Bunde, Physica A 295, 441 (2001)

17. J.W. Kantelhardt, S.A. Zschiegner, E. Koscielny-Bunde, S. Havlin, A. Bunde, H.E. Stanley, Physica A 316, 87 (2002) 
18. Y. Liu, P. Cizeau, M. Meyer, C. Peng, H. Stanley, Physica A 245, 437 (1997)

19. M. Ausloos, N. Vandewalle, Ph. Boveroux, A. Minguet, K. Ivanova, Physica A 274, 229 (1999)

20. M.A. Sánchez-Granero, J.E. Trinidad Segovia, J. García Pérez, Physica A 387, 5543 (2008)

21. E. Bayraktar, H.V. Poor, K.R. Sircar, Int. J. Theor. Appl. Finance 7, 615 (2004)

22. S.-H. Chen, T. Lux, M. Marchesi, J. Econ. Behav. Organ. 46, 327 (2001)

23. S. Mercik, K. Weron, Acta Physica Polonica B 34, 3773 (2003)

24. B.B. Mandelbrot, The Fractal Geometry of Nature (W.H. Freeman \& Company, New York, 1982)

25. C. Brown, L. Liebovitch, Fractal Analysis (SAGE Publications Inc., California, 2010)

26. C. Bandt, T. Retta, Fund. Math. 141, 257 (1992)

27. F.G. Arenas, M.A. Sánchez-Granero, Rend. Istit. Mat. Univ. Trieste, Suppl. XXX, 21 (1999)
28. F.G. Arenas, M.A. Sánchez-Granero, A characterization of self-similar symbolic spaces, Mediterr. J. Math., in press, doi: 10.1007/s00009-011-0146-4

29. M.A. Sánchez-Granero, M. Fernández-Martínez, Fractal dimension for fractal structures, preprint, arXiv:1007.3236v1 [nlin.CD] (2011)

30. M.A. Sánchez-Granero, Applications of fractal structures, in Proceedings of the Workshop in Applied Topology WiAT'10 (2010), pp. 1-10

31. K. Falconer, Fractal Geometry, Mathematical Foundations and Applications (John Wiley \& Sons Inc., Chichester, 1990)

32. M. Fernández-Martínez, M.A. Sánchez-Granero, Fractal dimension for fractal structures: A Hausdorff approach, Topol. Appl., in press,

doi: 10.1016/j.topol.2011.04.023

33. B.B. Mandelbrot, Gaussian self-affinity and fractals (Springer-Verlag, New York, 2002)

34. M. Maejima, Sugaku Expositions 2 1, 103 (1989) 